\title{
Giant intracranial aneurysm
}

\author{
James Scozzafava MD, Garnette Sutherland MD
}

Competing interests: None declared.

This article has been peer reviewed.

Affiliations: From the Medicine (Scozzafava), and the Division of Neurosurgery, Department of Clinical Neurosciences (Sutherland), University of Calgary, Calgary, Alta.

Correspondence to: Dr. James Scozzafava, jjs9@ualberta.ca

CMAJ 2011. DOI:10.1503 /cmaj.080184

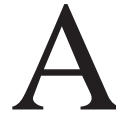
examination was not possible. Department of Critical Care 45-year-old man was brought to the emergency department by his family because of a change in personality and inappropriate behaviour that had progressed over several years. In the weeks before admission, he had become increasingly agitated, confused and lethargic. On examination, he was drowsy and displayed features of akinetic mutism. He had no spontaneous speech and stared blankly into space. He was covered in feces and unable to answer questions. The entire history was obtained from his family, and a detailed neurologic

Computed tomography (CT) and magnetic resonance imaging (MRI) of the brain showed a giant intracranial aneurysm (Figure 1A; see Appendix 1 for additional images, available at www.cmaj.ca/cgi/content/full/cmaj.080184/DC1), with vasogenic edema in the right frontal lobe. Maximal dimensions of the aneurysm were $6.3 \times$ $5 \mathrm{~cm}$. Conventional contrast cerebral angiography showed that the partially thrombosed aneurysm originated from the bifurcation of the

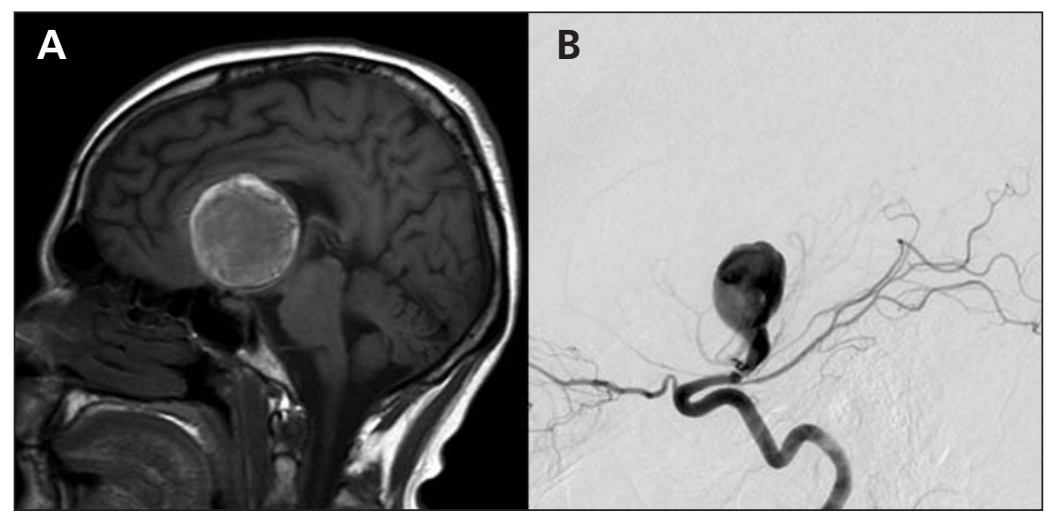

Figure 1: (A) Sagittal $T_{1}$ sequence magnetic resonance image of the head of a 45-year-old man, showing a large midline aneurysm compressing the thalamic and hypothalamic structures, as well as the superior anterior aspect of the brainstem. (B) Conventional cerebral angiogram showing a giant partially thrombosed aneurysm originating from the bifurcation of the right internal carotid artery. right internal carotid artery (Figure 1B). It also showed an incomplete circle of Willis with a virtually absent proximal right anterior cerebral artery and a small posterior communicating artery (see Appendix 1 for diagram illustrating the circle of Willis).

The patient was taken to the operating room, where temporary ligation of the extracranial internal carotid artery showed immediate cessation of pulsation within the aneurysm. A decision was made to obliterate the right extracranial internal carotid artery to preserve the orbitalophthalmic collateral artery. Clip occlusion of the extracranial internal carotid artery was performed, together with an extracranial to intracranial bypass. Intraoperative micro-Doppler studies showed adequate flow within the middle cerebral artery branches. (For more details on the surgical procedure, see Appendix 1.)

A postoperative CT angiogram showed obliteration and thrombosis of the aneurysm (Appendix 1). The patient's neurologic status improved progressively and, at the time of discharge, he was oriented to time, place and person, and was able to converse well and to carry out his usual activities. He returned to the workforce shortly after discharge and has maintained function over the three years since the procedure.

Giant intracranial aneurysms, defined as aneurysms greater than $25 \mathrm{~mm}$ in diameter, comprise about $5 \%$ of all cerebral aneurysms. Untreated, they carry a five-year mortality rate in excess of $80 \%$. In contrast, surgical and endovascular management of such aneurysms provide over $70 \%$ of patients with an excellent outcome. ${ }^{1,2}$

\section{References}

1. Drake CG. Giant intracranial aneurysms: experience with surgical treatment in 174 patients. Clin Neurosurg 1979;26:12-95.

2. Choi IS, David C. Giant intracranial aneurysms: development, clinical presentation and treatment. Eur J Radiol 2003;46:178-94 